\title{
Documenting New Ways of Delivering Care Under Oregon's Alternative Payment and Advanced Care Model
}

\author{
Erika K. Cottrell, PhD, MPP, Katie Dambrun, MPH, Jean O'Malley, MPH, \\ R. Lorie Jacob, ScM, Ned Mossman, MPH, Charles Ashou, and \\ Jobn Heintzman, MD, MPH
}

Background: The fee-for-service reimbursement system that dominates health care throughout the United States links payment to a billable office visit with a physician or advanced practice provider. Under Oregon's Alternative Payment and Advanced Care Model (APCM), initiated in 2013, participating community health centers (CHCs) received per-member-per-month payments for empaneled Medicaid patients in lieu of standard fee-for-service Medicaid payments. With Medicaid revenue under APCM no longer tied solely to the volume of visits, the Oregon Health Authority needed a way to document the full range of care and services that CHCs were providing to their patients, including nontraditional patient encounters taking place outside of traditional face-to-face visits with a billable provider. Toward this end, program leadership defined 18 visit and nonvisit-based care activities- "Care Services That Engage Patients" (Care STEPs) - that APCM CHCs were asked to document in the electronic health record to demonstrate continued empanelment.

Objective: To describe trends in rates of traditional face-to-face office visits and Care STEPs documentation among CHCs involved in the first 3 phases of APCM implementation.

Research Design: The study population included the 9 CHCs involved in the first 3 phases of APCM implementation. Using data from the electronic health record, quarterly summary rates for office visits and Care STEPs were calculated for the first 18 quarters of implementation (March 1, 2013 to June 30, 2017).

Results: Among participating CHCs, the mean rate of face-to-face visits with billable providers declined from $635 \pm 128$ to $461 \pm 109$ visits/1000 patients/quarter (mean difference, - 174; 95\% CI, - 255, -94). Care STEPs documentation increased from $831 \pm 174$ to $1017 \pm 369$ Care Steps/1000 patients/quarter, but the difference was not statistically significant. Care STEPs within the category of New Visit Types were documented most frequently. There were significant increases in documentation of Patient Care Coordination and Integration and a small, albeit significant, increase in Reducing Barriers to Health. There was a significant decline in the documentation of Care STEPs by physicians and advanced practice providers an increase in documentation by ancillary staff.

Conclusions: These findings suggest that APCM is increasing CHCs' capacity to experiment with new ways of providing care beyond the traditional face-to-face office visit with a physician or advanced practice provider. However, CHCs may choose different ways to change the delivery of care and some CHCs have implemented these changes more quickly than others. Future mixed-methods research is needed to understand barriers and facilitators to changing the delivery of care after APCM implementation. (J Am Board Fam Med 2021;34:78-88.)

Keywords: Capitation Fee, Community Health Centers, Health Care Systems, Health Policy, Medicaid, Oregon, Organizational Innovation, Patient-Centered Care, Primary Health Care, Workforce

\section{Introduction}

The concept of the Patient-Centered Medical Home $(\mathrm{PCMH})$ has been evolving since its inception, but is

This article was externally peer reviewed.

Submitted 17 January 2020; revised 14 September 2020; accepted 18 September 2020.

From the Department of Family Medicine, Oregon Health \& Science University, Portland, OR (EKC, JO, JH); OCHIN, Inc., Portland, OR (EKC, KD, JO, RLJ, NM); Virginia Garcia Memorial Health Center, Hillsboro, OR (CA). centered on the goals of "improving the health of whole people, families, communities and populations, and on increasing the value of health care." Many of

Funding: This work was supported by in part by a grant from the Robert Wood Johnson Foundation (RWJF 71125) and in part by the Agency for Healthcare Research and Quality (R01HS022651).

Conflict of interest: None.

Corresponding author: Erika K. Cottrell, $\mathrm{PhD}$, MPP, OCHIN, Inc., 1881 SW Naito Pkwy, Portland, OR 97201 (E-mail: cottrele@ohsu.edu). 
the core attributes of the PCMH model-including the provision of patient-centered, comprehensive, team-based, coordinated, and accessible care ${ }^{2}-$ require an expansion in the type of services that primary care clinics provide to patients, as well as flexibility and innovation in clinic staffing and reimbursement models. ${ }^{3,4}$ However, the fee-for-service reimbursement system that dominates health care throughout the US limits the ability of primary care clinics to fully implement the PCMH model by tying payment to a billable face-to-face office visit with a physician or advanced practice provider (APP). This challenge is especially acute in community health centers (CHCs) that serve vulnerable patient populations with a wide range of medical and social needs that may be difficult to address within the confines of a traditional 15 minute face-to-face visit. ${ }^{5,6}$ To overcome this challenge, starting in April of 2013, the Oregon Health Authority and the Oregon Primary Care Association (OPCA) launched the Alternative Payment and Advanced Care Model (APCM) in Oregon CHCs. Under APCM, CHCs receive per-member-per-month payments for empaneled Medicaid patients in lieu of standard feefor-service Medicaid payments. Removing the link between volume of face-to-face visits and clinic revenue was intended to align the payment structure with the PCMH goals of providing high-quality, team-based, patient-centered care. ${ }^{7}$ Three Oregon CHCs took part in the first phase of APCM implementation in 2013; by 2019, a total of 19 CHCs were participating in Oregon's APCM program. ${ }^{8}$

The agreement between participating $\mathrm{CHCs}$ and the Oregon Health Authority stated clearly that the APCM model was intended "to incent a significant transition in patient centered care," that would likely result in a "reduction in traditional, billable patient visits," and an "increase in nonbillable patient encounters." Participating CHCs were required to take part in quarterly Learning Community sessions, which provided a forum for sharing best practices and innovative models for transforming primary care under this new more flexible payment model. However, with Medicaid revenue under APCM no longer tied solely to billable patient visits, the Oregon Health Authority needed a way to understand the full range of care and services that CHCs were providing to their patients. Toward this end, the Oregon Health Authority and OPCA created a list of 18 specific Care and Services That Engage Patients (Care STEPs) to capture nonbillable, nonvisit-based care and services, defined as "direct interaction between health center staff and the patient, the patient's family or authorized representative(s) through either in-person, digital, group visits, or telephonic means." Care STEPs were grouped into 4 general categories: 1) New Visit Types, 2) Patient Care Coordination and Integration, 3) Education, Wellness, and Health Promotion, and 4) Reducing Barriers to Health. ${ }^{9}$ Although some Care STEPs had an existing standardized mechanism for documentation in the electronic health record (EHR), (eg, behavioral health screening), most of the Care STEPs required new means of documentation (eg, exercise class or support group participation). OPCA worked with the primary EHR vendor, OCHIN, to develop a tracking tool that would capture these additional Care STEPs. ${ }^{10}$ Participating $\mathrm{CHCs}$ were required to document Care STEPs in their EHR and to include this documentation in quarterly reports to the state., 911

Aggregate reporting to the state points to an overall increase in Care STEPs documentation and a decrease in traditional face-to-face visits since the beginning of APCM implementation. ${ }^{12}$ However, less is known about patterns of Care STEPs documentation over time or how these patterns may differ between CHCs. Findings from qualitative data collection among the CHCs participating in the first phase of APCM implementation (published elsewhere) highlighted a great deal of variation in both the consistency of Care STEPs documentation and in the awareness of the need for such documentation. ${ }^{13}$ Despite a consistent acknowledgment of the importance of team-based care and the need to accommodate alternative ways of providing care within the PCMH model, there were differences between $\mathrm{CHCs}$ and even between care teams within a single $\mathrm{CHC}$ with respect to their understanding of how APCM changed payment, the freedom this payment change afforded in terms of the types of care they could provide, and the priority placed on documenting Care STEPs. Documenting Care STEPs required changes in workflows, and CHC staff needed training on the types of activities requiring documentation as well as where and how to enter Care STEPs in the EHR. Moreover, many $\mathrm{CHC}$ staff expressed fatigue with the sheer number of documentation requirements, the evolving definitions of Care STEPs, and the imperative of keeping up with changes in EHR tools for capturing Care STEPs to meet state reporting requirements. ${ }^{13}$ 
Building on these early qualitative findings, the objective of this article was to describe trends in traditional face-to-face visits and Care STEPs documentation among $\mathrm{CHCs}$ involved in the first 3 phases of APCM implementation, both by category of Care STEPs and type of provider. We hypothesized that 1) traditional face-to-face visits would decline, 2) Care STEPs documentation would increase, 3) there would be variation between CHCs with respect to Care STEPs documentation, but this variation would decrease over the 3 phases of implementation. We also expected to see an increase in Care STEPs documented in the EHR by ancillary staff (providers other than physicians and APPs, whose visits were not billable under fee-for-service).

\section{Methods \\ Setting}

This study was approved by the Oregon Health \& Science University Institutional Review Board. The study population included the $9 \mathrm{CHCs}$ involved in the first 3 phases of APCM implementation. Three CHCs joined APCM in March 2013 (phase 1), with 3 additional CHCs joining in July 2014 (phase 2), and 3 more CHCs in July 2015 (phase 3). All CHCs were members of OCHIN, Inc., a health center-controlled network that hosts a centrally managed instance of Epic for a national network of CHCs serving over 2 million patients across 20 states. ${ }^{14-16}$

\section{Data and Analysis}

Data were extracted from the OCHIN EHR to calculate quarterly summary rates for traditional faceto-face office visits and Care STEPs over the first 18 quarters of APCM implementation (March 1, 2013 to June 30, 2017, with the first quarter limited to 1 month). We controlled for the shorter length of the first quarter by reporting all results as rates per 1000 attributed patient days. For each $\mathrm{CHC}$, data were extracted from the time of APCM entry to the end of the study period. We used the definition for traditional face-to-face visits as defined by the Uniform Data System requirements for Federally Qualified Health Centers, specified as "documented, face-to-face contacts between a patient and a provider who exercises independent professional judgment in the provision of services to the patient."17 EHR data were extracted for the 18 specific Care STEPs listed in Table 1, based on the definitions and guidance developed by OPCA and the Oregon Health Authority.
Rates per 1000 patients were calculated using the denominator of attributed patients reported to the Oregon Health Authority for each CHC in a given quarter. Rates of traditional face-to-face visits and Care STEPs were calculated by $\mathrm{CHC}$ and phase of implementation. Rates of Care STEPs documentation were also calculated separately for physicians and APPs (ie, medical doctors [MD], doctors of osteopathic medicine [DO], advanced practice nurses, nurse practitioners $[\mathrm{NP}]$, or physician assistants $[\mathrm{PA}]$ ), and ancillary staff (ie, medical assistants [MAs], social workers, registered nurse $[\mathrm{RN}]$, licensed practice nurse [LPN], and other support staff). Finally, rates per 1000 were calculated separately for each $\mathrm{CHC}$ by category of Care STEPs (ie, New Visit Type; Coordination and Integration; Education, Wellness, and Health Promotion; and Reducing Barriers to Health).

Mean differences in traditional face-to-face visit or Care STEPs rates between the first and last quarters of the observation period for each $\mathrm{CHC}$ were determined by paired $t$-test. Slopes were

Table 1. List of Non-Billable and Non-Visit-Based Care and Services (Care STEPs) for Documentation in Oregon's APCM Community Health Centers

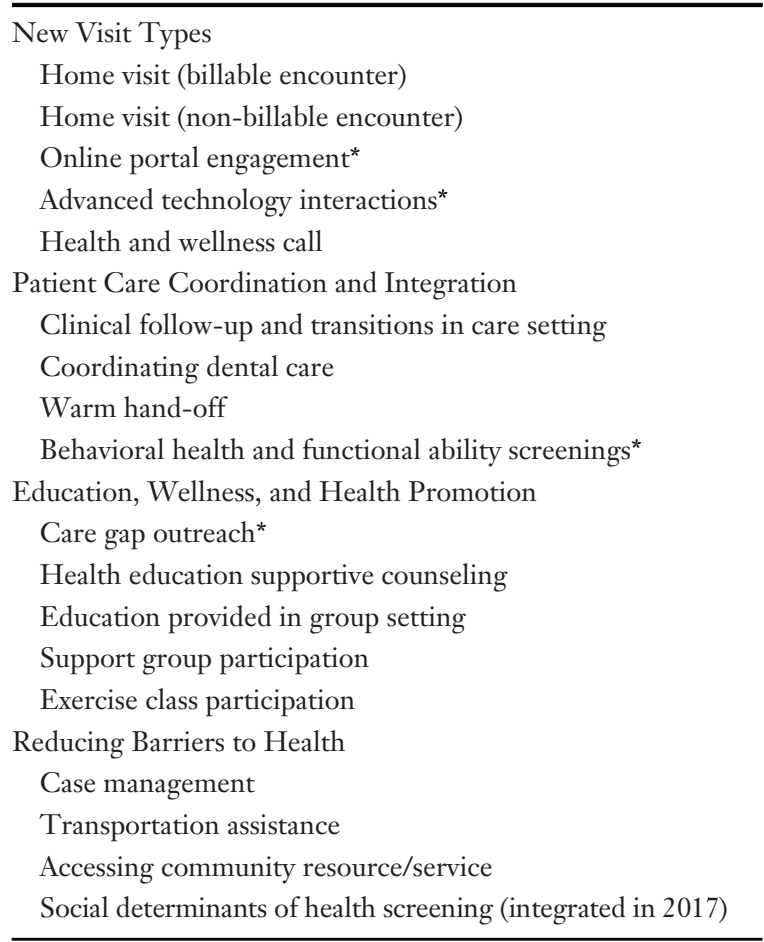

Care STEPs, care and services that engage patients; APCM, Alternative Payment and Advanced Care Model.

*Indicates that there was a standardized way for capturing in the electronic health record before APCM implementation. 
Table 2. Rates of Traditional Face-to Face Visits and Care STEPs Documentation in Oregon's APCM Community Health Centers (March 1, 2013 to June 30, 2017)

\begin{tabular}{lccc}
\hline Mean Rate/1000 Patients/Quarter (SD) & Start of Study Period & End of Study Period & Mean Difference (95\% CI) \\
\hline UDS traditional face-to-face visits per 1000 & $635(128)$ & $461(109)$ & $-174(-255,-94)^{*}$ \\
Care STEPs per 1000 (all providers) & $831(174)$ & $1017(369)$ & $186(-89,460)$ \\
Care STEPs per 1000 (physicians and APPs) & $279(133)$ & $221(156)$ & $-58(-162,45)$ \\
New visit types per 1000 & $688(168)$ & $675(193)$ & $-13(-131,105)$ \\
Care coordination per 1000 & $122(53)$ & $266(179)$ & $145(11,279)^{*}$ \\
Education/wellness per 1000 & $7(9)$ & $42(68)$ & $35(-19,89)$ \\
Barriers to health per 1000 & $14(19)$ & $33(39)$ & $19(-10,49)$ \\
\hline
\end{tabular}

*Statistically significant.

Care STEPs, Care and Services That Engage Patients; APCM, Alternative Payment and Care Model; UDS, Uniform Data Service; APP, advanced practice provider; CI, confidence interval.

calculated using generalized linear mixed models of the rates over time adjusting for within CHC correlation using robust sandwich estimators. Slopes were stratified by phase of APCM implementation, with 3 CHCs per phase. The period of observation was longer for CHCs in the earlier phases of APCM implementation (phase 1: 18 quarters; phase 2: 12 quarters; phase 3: 8 quarters).

\section{Results}

As expected, we observed a significant decrease in the rate of traditional face-to-face visits after APCM implementation, from a mean of 635 (SD, 128) visits per 1000 patients in the entry quarters of APCM implementation, to 461 (SD, 109) visits per 1000 patients in the final quarter of the study period (Table 2). Rates of decline in traditional face-to-face visits increased with each phase of implementation, with an average decline of 10.8 visits/1000 patients/ quarter (95\% CI, -17.8, -3.7) among phase 1 CHCs, 18.7 visits/1000 patients/quarter among phase 2 CHCs $(95 \% \mathrm{CI},-32.21,-5.1)$ and 27.7 among phase $3 \mathrm{CHCs}(95 \% \mathrm{CI},-31.6,23.8)$ (Figure 1). However, there was variability in the rate of decline between CHCs within each phase, with some

Figure 1. Quarterly Rates of Traditional Face-to-Face Visits, Physicians and Advanced Practice Providers, CHCs in Phases 1 to 3 of Oregon's APCM Program (March 1, 2013 to June 30, 2017). Abbreviations: APCM, Alternative Payment and Advanced Care Model; CHC, Community Health Center; UDS, Uniform Data Service.

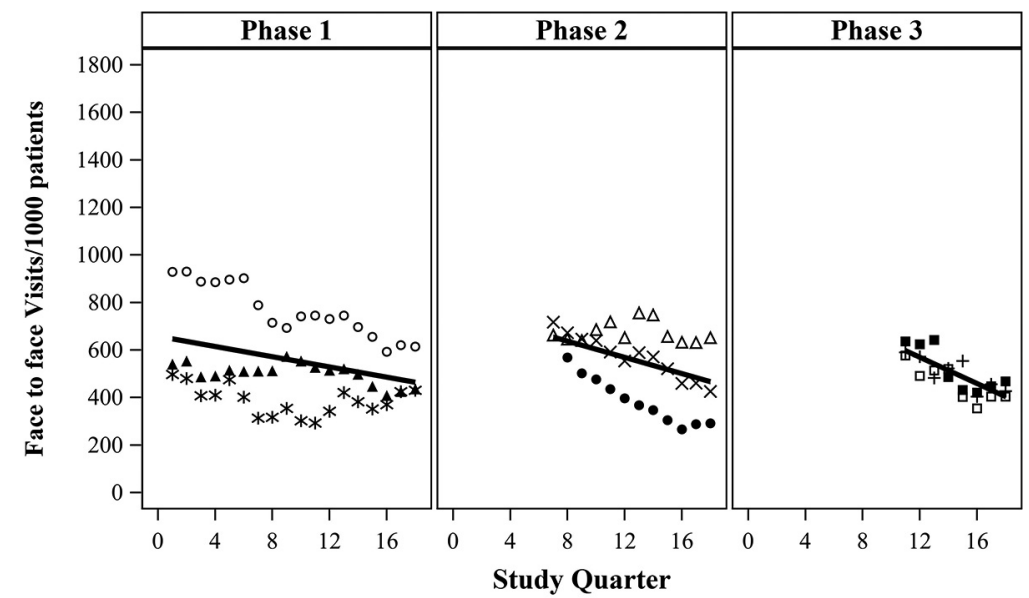

Lines are trend lines from regression analysis. Marker styles indicate individual Community Health Centers. Slopes represent the change in UDS visits $/ 1000$ patients/quarter $(95 \% \mathrm{CI})$, stratified by phase of implementation, with $3 \mathrm{CHCs}$ in each phase: Phase 1 (March 1, 2013 to June 30, 2017): -10.8 (-17.8, -3.7 ); Phase 2 (July 1, 2014 to June 30, 2017): -18.7 (-32.2, -5.1); Phase 3 (July $1,2015$ to June 30,2017$)$ : $-27.7(-31.6,-23.8)$. 
Figure 2. Quarterly Rates of Care STEPs Documentation (All Categories), All Providers, CHCs in Phases 1 to 3 of Oregon's APCM Program (March 1, 2013 to June 30, 2017). Abbreviations: Care STEPs, Care and Services That Engage Patients; APCM, Alternative Payment and Advanced Care Model; CHC, Community Health Center.

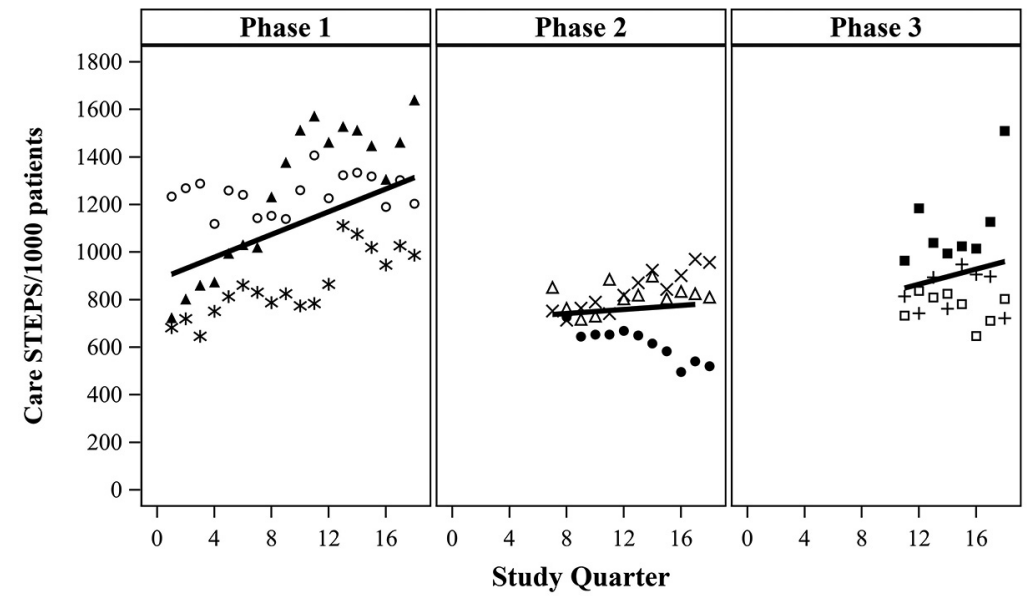

Lines are trend lines from regression analysis. Marker styles indicate individual Community Health Centers. Slopes represent the change in Care STEPs/1000 patients/quarter ( $95 \%$ Confidence Interval), stratified by phase of implementation, with 3 CHCs per phase: Phase 1 (March 1, 2013 to June 30, 2017): 23.0 (0.0, 47.9); Phase 2 (July 1, 2014 to June 30, 2017): 4.3 (-14.2, 22.7);

Phase 3 (July 1, 2015 to June 30, 2017): 16.0 (-25.1, 57.1).

CHCs exhibiting a steady decline over the period of observation and others fluctuating or remaining relatively stable. This was especially true for the CHCs in the first phase of implementation, where there was a marked decrease in face-to-face visits (from 939 per 1000 patients to 621 per 1000) in $1 \mathrm{CHC}$, but a more modest overall decline, and a decrease followed by a subsequent increase, in the other 2 CHCs. With

Figure 3. Quarterly Rates of Care STEPs Documentation (All Categories), Physicians and Advanced Practice Providers, CHCs in Phases 1 to 3 of Oregon's APCM Program (March 1, 2013 - June 30, 2017). Abbreviations: Care STEPs, Care and Services That Engage Patients; APCM, Alternative Payment and Advanced Care Model; CHC, Community Health Center.

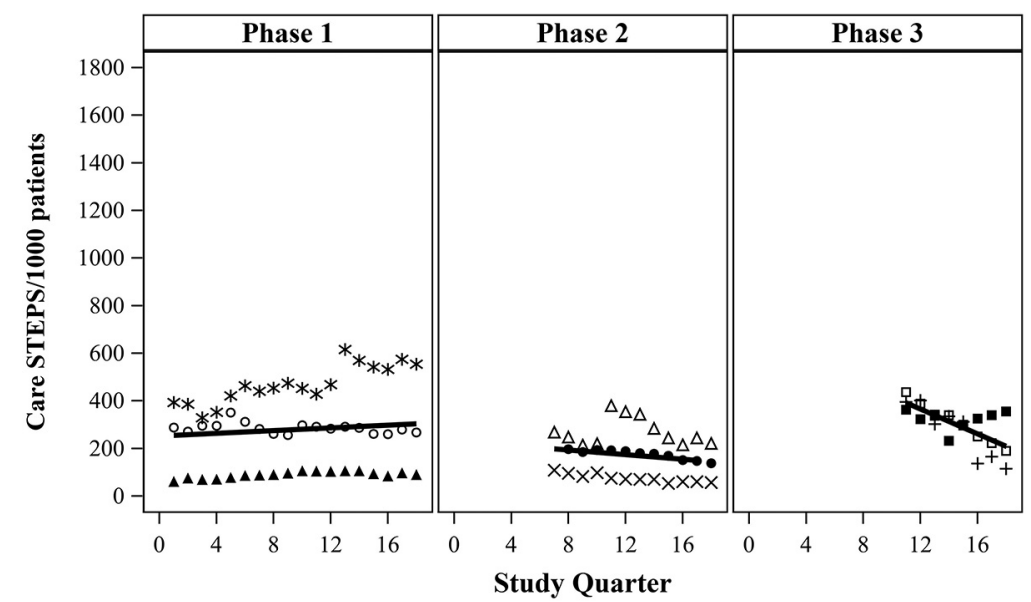

Lines are trend lines from regression analysis. Marker styles indicate individual Community Health Centers. Slopes represent the change Care STEPs/1000 patients /quarter documented by physicians and APPs (95\% Confidence Interval), stratified by phase of implementation, with 3 CHCs per phase: Phase 1 (March 1, 2013 to June 30, 2017): 2.8 (-3.2, 8.8); Phase 2 (July 1, 2014 to June 30, 2017): $-4.9(-6.1,-3.7)$; Phase 3 (July 1,2015 to June 30,2017$)$ : $-26.1(-47.8,4.4)$. 
Figure 4. Quarterly Rates of Care STEPs Documentation (New Visit Types), All Providers, CHCs in Phases 1 to 3 of Oregon's APCM Program (March 1, 2013 - June 30, 2017). Abbreviations: Care STEPs, Care and Services That Engage Patients; APCM, Alternative Payment and Advanced Care Model; CHC, Community Health Center.

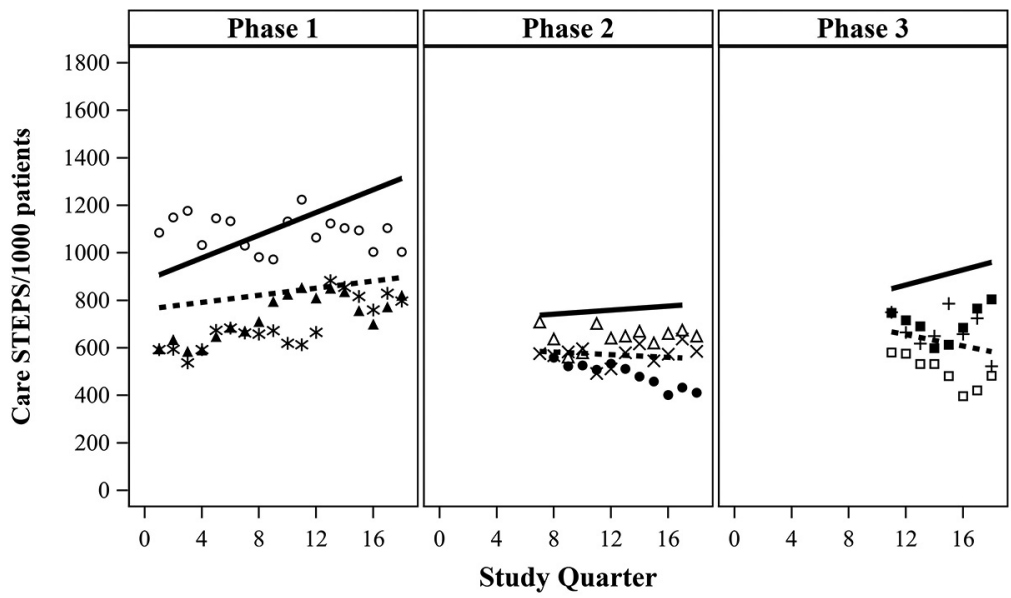

Solid lines are trend lines for total Care STEPS. Dashed lines are trend lines for New Visit Types. Marker styles indicate individual Community Health Centers. Slopes represent the change in New Visit Types Care STEPs/1000 patients/quarter (95\% Confidence Interval), stratified by phase of implementation, with 3 CHCs per phase: Phase 1 (March 1, 2013 to June 30, 2017): 7.5 (-3.2, 18.1); Phase 2 (July 1, 2014 to June 30, 2017): $-2.8(-10.6,5.0)$; Phase 3 (July 1, 2015 to June 30, 2017): $-12.0(-28.0,4.1)$.

the exception of $1 \mathrm{CHC}$ in phase 2 that had a small increase in face-to-face visits and then leveled off, the $\mathrm{CHCs}$ in phases 2 and 3 exhibited a consistent downward trend in rates of face-to-face visits (Figure 1).

Along with the decrease in traditional face-toface visits, we observed an increase in overall Care STEP documentation, from a mean of 831 (SD, 174) per 1000 patients in the entry quarter of APCM implementation to a mean of 1017 (SD, 369) per 1000 patients in the final quarter of the study period (Table 2). Phase 1 CHCs had the longest observation period (18 quarters) and showed a marginally significant rate of increase in Care STEPs documentation (average rate of increase of 23.9 per 1000 patients per quarter; $95 \% \mathrm{CI},-0.01$, 47.9). The linear trends in phase 2 and phase 3 CHCs were not statistically significant. When looking at rates broken down by $\mathrm{CHC}$, there was variation between CHCs across all phases with respect to the overall number and rate of change in Care STEPs documented. For example, in phase 1, 1 CHC doubled their Care STEPs documentation over the study period (from 722 to 1638 per 1000 patients/quarter), with another exhibiting a more modest increase, and the third showing a slight decline. In phase 2, 2 of the CHCs had fairly stable rates of Care STEPs documentation, while another decreased sharply (from 727 to 521 per 1000 patients). The trends among phase 3 CHCs were also varied, with all $3 \mathrm{CHCs}$ showing increases or decreases in documentation rates that were not maintained over time (Figure 2).

When comparing the rates of Care STEPs documentation by type of provider, there was a general decrease in Care STEPs documentation by physicians and APPs (Table 2), accompanied by an increase in documentation by ancillary providers across the phases of implementation (data not shown). The rate of Care STEPs documentation by physicians and APPs remained relatively stable for phase $1 \mathrm{CHCs}$, but declined significantly in phases 2 and 3, with an average decline of 4.9 Care STEPs per 1000 patients/ quarter $(95 \% \mathrm{CI},-6.1,-3.7)$ in phase 2 and 26.1 Care STEPs per 1000 patients/quarter (95\% CI, $-47.9,-4.4$ ) in phase 3 (Figure 3).

Overall, and across all 3 phases, rates of Care STEPs documentation were highest for activities categorized as New Visit Types (Table 2, Figure 4), followed by Coordination and Integration (Table 2, Figure 5). There were much lower rates of documentation in the categories of Education, Wellness and Health Promotion (Table 2, Figure 6) and 
Figure 5. Quarterly Rates of Care STEPs Documentation (Patient Care Coordination and Integration), All Providers, CHCs in Phases 1 to 3 of Oregon's APCM Program (March 1, 2013 to June 30, 2017). Abbreviations: Care STEPs, Care and Services That Engage Patients; APCM, Alternative Payment and Advanced Care Model; CHC, Community Health Center.

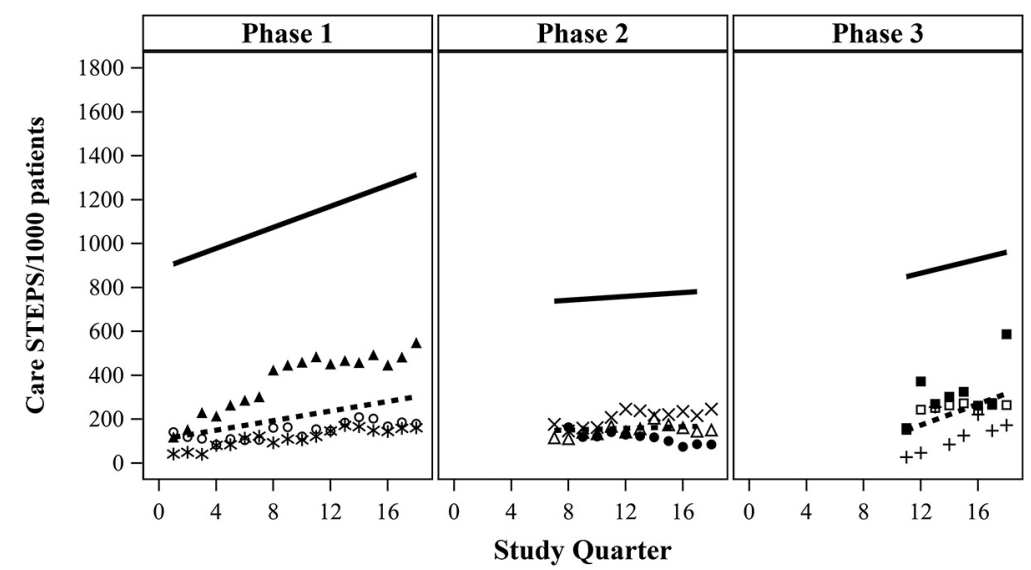

Solid lines are trend lines for total Care STEPS. Dashed lines are trend lines for Patient Care Coordination Care STEPS. Marker styles indicate individual Community Health Centers. Slopes represent the change in Patient Care Coordination and Integration Care STEPs/1000 patients/quarter (95\% Confidence Interval), stratified by phase of implementation, with 3 CHCs per phase: Phase 1 (March 1, 2013 to June 30, 2017): 10.9 (1.7, 20.0); Phase 2 (July 1, 2014 to June 30, 2017): -1.8 (-4.4, 8.0); Phase 3 (July 1, 2015 to June 30, 2017): 24.1 (7.9, 40.4).

Reducing Barriers to Health (Table 2, Figure 7). With respect to the rate of change within the different phases, there were significant increases in documentation of Coordination and Integration among the 3 CHCs implementing in phase 1 (increase of 10.9 per 1000 patients/quarter; $95 \% \mathrm{CI}, 1.7,20.0$ ) and phase 3 (increase of 24.1 per 1000 patients/ quarter; 95\% CI, 7.9, 40.4; Figure 5), and a small, albeit significant, increase in the documentation of Reducing Barriers to Health among the CHCs implementing in phase 3 (increase of 4.1 per 1000 patients/quarter; 95\% CI, 0.2, 8.1; Figure 7). All others were not significant (Figure 4, Figure 5, Figure 6, Figure 7).

\section{Discussion}

Many have argued that successful execution of the PCMH model requires a change in the way clinics are reimbursed to provide more patient-centered, team-based, and comprehensive patient care. ${ }^{18-20}$ The APCM per-member per-month model provides one mechanism for paying $\mathrm{CHCs}$ for care and services that traditional models of payment do not cover. By removing the linkage between volume of visits and clinic revenue, Oregon's APCM pilot was intended to encourage clinics to implement innovative models of care and to use different members of the care team to deliver the care that patients need, when they need it. This does not always mean a face-to-face visit with a physician or APP. Indeed, it was a stated expectation that CHCs taking part in APCM may see a decline in traditional face-to-face visits with billable providers as they experimented with alternative ways of providing care to their patient populations. ${ }^{11}$ For example, in some cases, care can be provided over the phone or via the patient portal (eg, answering patient questions about medication side effects or going over lab results), provided by another team member (eg, a community health worker may be able to help patients find transportation resources or other social services), and/or patients can attend group sessions (eg, prenatal care, diabetes education, group exercise classes). ${ }^{13}$ All CHCs who participated in Oregon's APCM were expected to take part in quarterly Learning Community sessions where they explored innovative models for provding care and services outside of the traditional faceto-face visit. ${ }^{11,21}$ In addition, all CHCs were required to document these alternative ways of providing care-or Care STEPs—in their EHR and to 
Figure 6. Quarterly Rates of Care STEPs Documentation (Education, Wellness and Health Promotion), All Providers, CHCs in Phases 1 to 3 of Oregon's APCM Program (March 1, 2013 to June 30, 2017). Abbreviations: Care STEPs, Care and Services That Engage Patients; APCM, Alternative Payment and Advanced Care Model; CHC, Community Health Center.

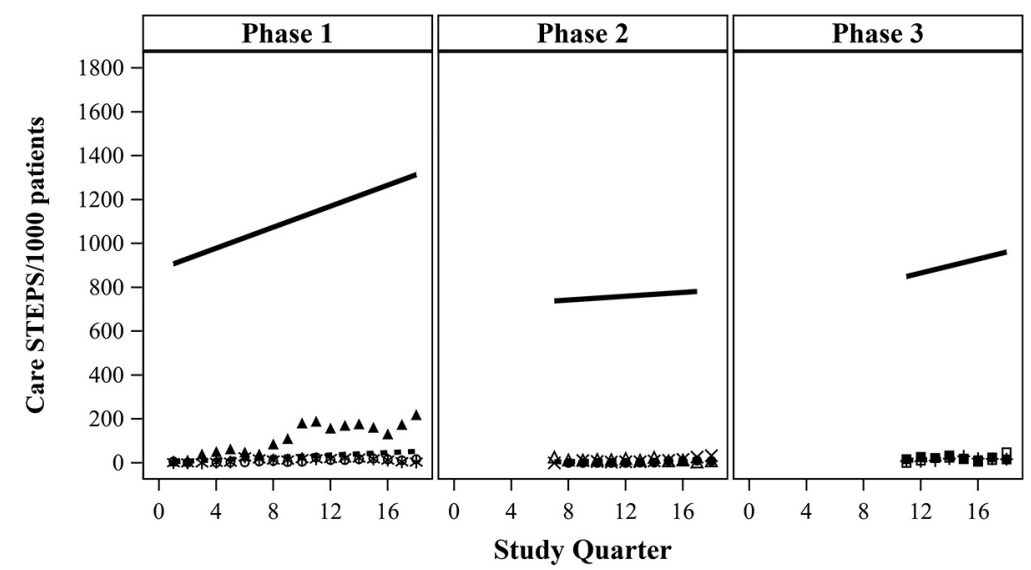

Solid lines are trend lines for total Care STEPS. Dashed lines are trend lines for Education, Wellness and Health Promotion Care STEPS. Marker styles indicate individual Community Health Centers. Slopes represent the change in Education, Wellness and Health Promotion Care STEPs/1000 patients/quarter (95\% Confidence Interval), stratified by phase of implementation, with 3 CHCs per phase: Phase 1 (March 1, 2013 to June 30, 2017): $4.5(-1.5,10.6)$; Phase 2 (July 1, 2014 to June 30, 2017): 0.9 (-0.9, 2.7); Phase 3 (July 1, 2015 to June 30, 2017): $2.1(-0.6,4.9)$.

provide quarterly reports to the Oregon Health Authority. Previous studies investigating the first phase of APCM implementation in Oregon suggested that participating $\mathrm{CHCs}$ were able to maintain or even improve some metrics. For example, compared to CHCs that did not take part in APCM, participating $\mathrm{CHCs}$ exhibited stable levels of primary care access, an increase in the availability of same day apointments, ${ }^{22}$ and a decline in traditional primary care services such as imaging among APCM, relative to non-APCM, CHCs. ${ }^{23}$ This study builds on these findings by assessing patterns of traditional face-toface visits and Care STEPs documentation over the first 3 phases of APCM implementation.

Analysis of EHR data among CHCs taking part in the first 3 phases of APCM implementation confirmed our hypothesis that there would be a decline in rates of traditional face-to-face visits. The rate of decline was significant and increased across all 3 phases, with less variation between CHCs over the course of the observation period. Documentation of Care STEPs increased among CHCs within all 3 phases of implementation. This increase was marginally significant among Phase $1 \mathrm{CHCs}$, who had the longest period of implementation, but not significant in Phases 2 and 3. Moreover, patterns of
Care STEPs documentation varied widely between $\mathrm{CHCs}$, with some $\mathrm{CHCs}$ showing rapid increases in documentation, and others exhibiting up-anddown trends or little change over the observation period. This supports findings from an earlier qualitative study, which suggested that providers and staff needed time to adapt to the new workflows required for documenting Care STEPs, and that some CHCs placed more emphasis on implementing the workflow changes needed to both deliver and document Care STEPs. ${ }^{13}$ Finally, as hypothesized, there was a steady and significant decrease in the rate of Care STEPs documentation by physicians and other APPs, especially in the latter phases of APCM implementation, suggesting that APCM is enabling other team members to take a bigger role in effectively and efficiently delivering care.

With respect to the different categories of Care STEPs, New Visit Types and Care Coordination were documented at much higher rates than Reducing Barriers to Health or Education, Wellness and Health Promotion. Although there were significant increases in documentation rates for Care Coordination activities in phases 1 and 3 and Reducing Barriers to Health in phase 3, changes in other categories were not significant. There are 
Figure 7. Quarterly Rates of Care STEPs Documentation (Reducing Barriers to Health), All Providers, CHCs in Phases 1 to 3 of Oregon's APCM Program (March 1, 2013 to June 30, 2017). Abbreviations: Care STEPs, Care and Services That Engage Patients; APCM, Alternative Payment and Advanced Care Methodology; CHC, Community Health Center.

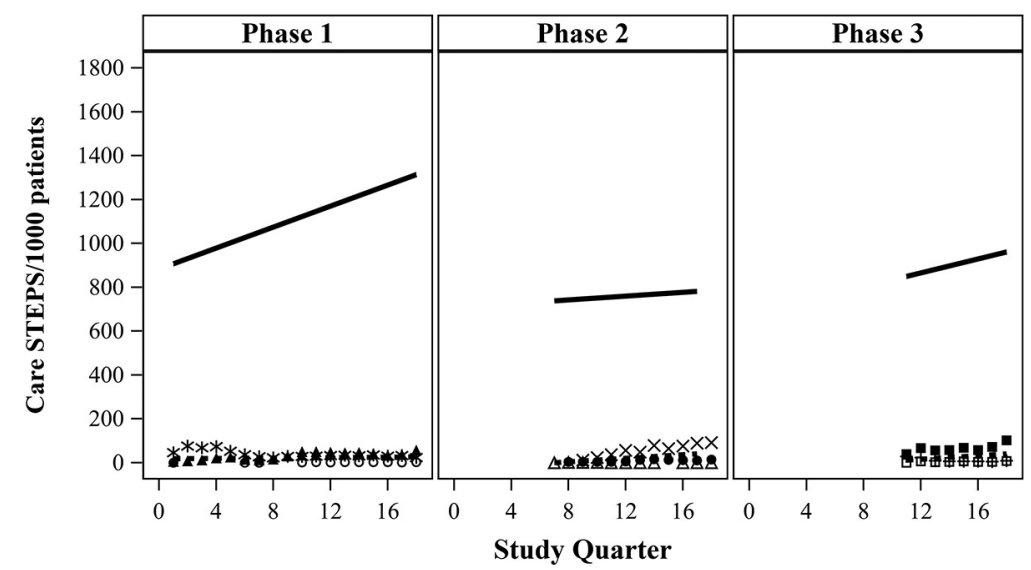

Solid lines are trend lines for total Care STEPS. Dashed lines are trend lines for Rdeucing Barriers to Health Care STEPS. Marker styles indicate individual Community Health Centers. Slopes represent the change in Reducing Barriers to Health Care STEPs/1000 patients/quarter (95\% Confidence Interval), stratified by phase of implementation, with 3 CHCs per phase: Phase 1 (March 1, 2013 to June 30, 2017): 0.4 (-1.8,2.6); Phase 2 (July 1, 2014 to June 30, 2017): 3.6 (-1.6, 8.8); Phase 3 (July 1, 2015 to June 30, 2017): $4.1(0.2,8.1)$.

several potential explanations for these differences. First, some activities within New Visit Types and Care Coordination (eg, online portal visits, advanced technology interactions, behavioral health screening) had a systematic mechanism for EHR documentation in place before the beginning of APCM, and thus may have been easier to document. Second, although Care Coordination activities (eg, clinical follow-up, coordinating care) may not have had a systematic mechanism for EHR documentation, these activities were within the traditional scope of work for primary care practices and health center staff before APCM and there were likely established workflows and staffing models for providing these services. This may account for the significant increases in these activities among CHCs implementing in phases 1 and 3. Third, CHCs taking part in APCM were encouraged to focus on delivering and documenting nonvisit-based services that have traditionally fallen outside of the scope of clinical care, but are important to patient health, such as reducing barriers to health like transportation, screening for social determinants of health, or helping patients access social services. Not unexpectedly, the overall number of Care STEPs documented for Reducing Barriers to Health and Education, and
Wellness and Health Promotion-categories that encompass these nontraditional primary care services-were low across all phases of implementation. However, there was a small increase in Reducing Barriers to Health documentation among the CHCs implementing in phases 2 and 3; this increase was significant among the $3 \mathrm{CHCs}$ in phase 3 , despite the shorter interval they had in which to implement changes. This could be due to clinics in later phases receiving more training and clarity about what types of activities should be documented as Care STEPs, as well as the increased focus on social determinants of health screening ( 1 of the activities under Reducing Barriers to Health) within the APCM Learning Community.

These preliminary descriptive findings suggest that APCM does enable clinics to experiment with alternative types of care and to use different types and levels of CHC staff to deliver care. However, these findings also highlight that change does not happen overnight and not all CHCs implement changes in the same way or at the same pace. Documenting Care STEPs is a new activity that may require additional training and/or changes to staffing and workflows. Differences between CHCs 
with respect to the categories of Care STEPs documented could be an indication that clinics are leveraging this new payment structure in diverse ways; these differences could reflect differing clinic priorities in relation to the populations they serve or the availability of staff with training and expertise in delivery of new types of services (eg, group exercise classes, community gardens). Future mixed-methods research is needed to understand rates of documentation over time as well as barriers and facilitators related to the delivery and documentation of different categories of Care STEPs.

This study has several limitations. First, we were only able to measure changes that were documented in the EHR. Thus, rates of documentation presented may underestimate the actual rates of Care STEP delivery. Second, our study period was limited to the first 18 quarters of APCM implementation (through June 2017). Although our analyses revealed a great deal of variation between CHCs during this first phase of implementation, it is likely that this variation will naturally begin to decline in later phases as clinics become more accustomed to the new reporting requirements and as lessons are passed along from the CHCs participating in earlier phases. Moreover, changing state reporting requirements may begin to bring documentation patterns among CHCs into greater alignment. Initially, participating $\mathrm{CHCs}$ were required to document at least 1 meaningful Care STEP within the past 12 months for $70 \%$ to $75 \%$ of patients. However, as of July 2017, the Oregon Health Authority shifted their policy, such that they would remove patients from APCM lists if they did not have a visit or Care STEP in an 8-quarter lookback period, resulting in no payment for those patients. Future studies should investigate the impact of this policy change on the rates of Care STEPs documentation after 2017. Perhaps more importantly, as Care STEPs documentation becomes more consistent, a priority for future research will be to understand whether changes in how care and services are delivered leads to any appreciable differences in patient outcomes.

\section{Conclusions}

Successful execution of the PCMH model may require a change in the way clinics are reimbursed to provide more comprehensive and patient-centered care. The APCM per-patient per-month model provides a mechanism for paying $\mathrm{CHCs}$ for care coordination and alternative care delivery services that traditional models of payment cannot cover. Analysis of EHR data from CHCs across the first 3 phases of APCM implementation showed a significant decline in rates traditional face-to-face visits. There was also an overall increase in Care STEPs documentation, although the rate of change was significant only among Phase $1 \mathrm{CHCs}$ who implemented APCM earliest and had the longest period of observation. Moreover, there was a great deal of variation in Care STEPs documentation between CHCs, suggesting that although APCM does enable clinics to experiment with providing alternative types of care, CHCs may choose different ways to change the delivery of care, and some $\mathrm{CHCs}$ are able to implement these changes more quickly than others. Finally, there was a significant decline in the delivery of Care STEPs by physicians and APPs, and an increase in delivery by ancillary staff, suggesting that APCM is increasing CHCs' capacity to deliver team-based care, which is a central tenet of the PCMH model.

The authors acknowledge the Alternative Payment and Care Methodology Program (APCM) Community Health Centers who allowed us into their practices to learn about their work and helped us to interpret our findings. The authors also thank the Oregon Health Authority and Oregon Primary Care Association who provided guidance in our methodology, interpretation of findings, and allowed us to learn from their implementation process for the APCM Program.

To see this article online, please go to: http://jabfm.org/content/ 34/1/78.full.

\section{References}

1. Stange KC, Nutting PA, Miller WL, et al. Defining and measuring the patient-centered medical home. J Gen Intern Med 2010;25:601-12.

2. Patient-Centered Primary Care Collaborative. Defining the medical home: a patient-centered philosophy that drives primary care excellence. Available from: https://www.pcpcc.org/about/medical-home.

3. Gelmon S, Bouranis N, Sandberg B, Petchel S. Strategies for addressing the challenges of patient-centered medical home implementation: lessons from Oregon.J Am Board Fam Med 2018;31:334-41.

4. Rissi JJ, Gelmon S, Saulino E, Merrithew N, Baker R, Hatcher P. Building the foundation for health system transformation: Oregon's Patient-Centered Primary Care Home program. J Public Health Manag Pract 2015;21:34-41.

5. Shin P, Rosenbaum S, Paradise J. Community health centers: the challenge of growing to meet the 
need for primary care in medically underserved communities. Kaiser Commission on Medicaid and the Uninsured. 2012 (Issue Paper No. 8098-02).

6. Proser M, Bysshe T, Weaver D, Yee R. Community health centers at the crossroads: growth and staffing needs. JAAPA 2015;28:49-53.

7. Hostetler C, Sisulak L, Cottrell EK, Arkind J, Likumahuwa S. Origins in Oregon: the alternative payment methodology project. 2014. Available from: https://www.healthaffairs.org/do/10.1377/hblog2014 0414.038476/full/.

8. Oregon Primary Care Association. Alternative payment \& advanced care model. Available from: https://www.orpca.org/initiatives/alternative-caremodel. Accessed October 15, 2019.

9. Oregon Primary Care Association. APCM Care STEPs report: care and services that engage patients. 2017. Available from: https://www.nashp. org/wp-content/uploads/2017/09/OPCA-APCMCare-STEPs-FINAL-8.2.17.pdf.

10. National Association of Community Health Centers. Spotlight on health center payment reform: Oregon alternative payment and advanced care model. December 2016. Available from: http://www.nachc. org/wp-content/uploads/2017/08/Oregon-FQHCAPM-December-2017.pdf.

11. Oregon Health Authority. Participation agreement for Oregon's alternative payment and care methodology (APCM). 2016. Available from: https://www. nashp.org/wp-content/uploads/2017/09/OregonPrimary-Care-Association-APM-ACM-PartnerAgreement-07062016.pdf.

12. Oregon Primary Care Association. Care STEPs: from documentation to care redesign. Portland, OR: Advanced Care Learning Community; 2018.

13. Cottrell EK, Hall JD, Kautz G. Reporting from the front lines: implementing Oregon's alternative payment methodology in federally qualified health centers. J Ambul Care Manage 2017;40:339-46.

14. Devoe JE, Gold R, Spofford M, et al. Developing a network of community health centers with a common electronic health record: description of the safety net west practice-based research network (SNW-PBRN). J Am Board Fam Med 2011;24:597-604.

15. DeVoe JE, Likumahuwa S, Eiff MP, et al. Lessons learned and challenges ahead: report from the OCHIN safety net west practice-based research network (PBRN). J Am Board Fam Med 2012; 25:560-4.

16. Devoe JE, Sears A. The OCHIN community information network: bringing together community health centers, information technology, and data to support a patient-centered medical village. J Am Board Fam Med 2013;26:271-8.

17. Bureau of Primary Health Care. UDS reporting instructions for health centers. 2014. Available from: https://bphc.hrsa.gov/sites/default/files/bphc/ datareporting/reporting/2014udsmanual.pdf.

18. Guterman S, Davis K, Stremikis K, Drake H. Innovation in Medicare and Medicaid will be central to health reform's success. Health Aff (Millwood) 2010;29:1188-93.

19. American College of Physicians. A system in need of change: restructuring payment policies to support patient-centered care. Position Paper. Philadelphia, PA: American College of Physicians; 2006.

20. McConnell KJ. Investing in primary care and dismantling fee-for-service. Milbank Quarterly 2019; 97:636-40.

21. Oregon Primary Care Association. Advanced care learning community. 2019. Available from: https:// www.orpca.org/initiatives/alternative-care-model/265apcm-learning-session-events. Accessed December 12, 2019.

22. Heintzman J, Cottrell EK, Angier H, et al. of Alternative payment methodology on primary care visits and scheduling. J Am Board Fam Med 2019;32:539-49.

23. Lindner S, Kaufman MR, Marino M, et al. A Medicaid alternative payment model program in Oregon led to reduced volume of imaging services. Health Aff (Millwood) 2020;39:1194-201. 